\title{
OPTIMIZATION OF ROUTING PROTOCOLS FOR SUPPORTING QOS OVER MOBILE AD HOC NETWORKS (MANET)
}

\author{
M. K. Ahmed, O. M. El-Ghandour, H. R. Solima \\ Helwan University, Electronics \& Communications and Computer \\ Engineering Department, Cairo, Egypt. \\ osamaghn@hotmail.come-mail: \\ prof-MK@hotmail.com e-mail: \\ hedia_ramadan@yahoo.come-mail:
}

(Received September 14, 2006 Accepted January 13, 2007)

Wireless Ad Hoc networks are relatively new and are gaining ground in research due to promises they offer. Wireless Ad hoc networks do not require predefined configuration and have no fixed infrastructure. They are selforganizing and self-configuring networks. Several protocols have been developed that vary in the performance and complexity. Most routing protocols for mobile ad hoc networks, such as: Ad Hoc On Demand Distance Vector Protocol (AODV), Dynamic Source Routing (DSR) are designed without explicitly considering quality of service of the generated route. These routing protocols provide the capability for establishing minimum hop paths between nodes on a best effort basis regardless of QoS. There is a new proposed scheme named Hierarchical Dynamic Source Routing (HDSR) supporting Qos over mobile ad hoc network (MANET) has been attracting significant current research interest. In our work, we evaluate the aspects of Qos concerning this protocol compared to the other schemes. The performance aspects we study are fraction of routing overhead, end-to-end delay, total capacity, MAC control packet capacity and throughput. We have shown that distribution of Forward Nodes (FN) in the network is important for optimization of the performance figures. We present an efficient and adaptive FN selection mechanism for HDSR as well as optimization of number of FNs in a given scenario. 
KEYWORDS: Ad hoc, AODV, DSR, HDSR, QoS, FN.

\section{INTRODUCTION}

Mobile ad hoc networking is becoming increasingly popular as a mean of providing instant networking to groups that may be within the transmission range of one another. These networks are self-initializing, self-configuring, and self-maintaining, all of which can be coined with term "self-organizing". Since connectivity changes constantly, a major challenge in mobile ad hoc network environments is a reliable and efficient routing service. Each node in the network acts as a router, forwarding data packets for other nodes. So, Routing is an essential part of network protocols to provide self-organizing capability, and it is the most widely studied element for ad hoc networks. A central challenge in the design of ad hoc networks is the development of dynamic routing protocols that can efficiently find routes between two communicating nodes. The routing protocol must be able to keep up with the high degree of node mobility that often changes the network topology drastically and unpredictably.

Various constraints are introduced by the Ad hoc networks:

-Dynamic topology: which evolves very quickly because each node can move arbitrarily and disappear randomly without any notification. From where need for routing mechanism which adapts with the nodes connectivity at a given moment is evident.

-Radio channel of communication: indeed the connections are with variable rates and limited bandwidth.

-Nodes function with batteries: a reduced autonomy in term of energy. Moreover each node serves as a host as well as a router and uses consequently its own energy to route flows intended for other nodes of the networks.

-Limited security: since ad hoc networks are more vulnerable to physical security threats, provisions for security must be made.

The ability to provide an adaptive quality of service (QoS) in such a mobile environment is a key to the success of next generation wireless communications systems. Recently there has been a considerable amount of QoS research. However, the main part of this research has been in the context of framework components, and much less progress has been made in addressing the issue of a group management to provide 
QoS within an ad hoc network.

\subsection{Ad Hoc Routing Protocols Overview}

Below we present an overview of representative ad hoc routing protocols. For the evaluated protocols, more extensive descriptions are provided in Section 3.

\subsubsection{Proactive Protocols}

They are also known as state-based/table driven protocols. Protocols that fall in this category perform periodic route table exchanges and continuously attempt to maintain a complete topological view of the network at each node. Hence, routes are readily available when data need to be sent.

\subsubsection{Link State}

Fisheye State Routing [1]. The amount of link state information received depends on the distance from the source. Nodes exchange link state for distant nodes with lower frequency than for nodes within a specified scope. Correctness is maintained due to the fact that routing information becomes more accurate as it is forwarded towards the destination.

Optimized Link State Routing [2]. Each node selects a set of its neighbors to be its Multipoint Relay MPR nodes. Link state information regarding this node is periodically transmitted only by its MPRs. MPRs provide an efficient method for flooding control packets. MPRs calculate shortest paths for their selectors and are used to form routes to every destination.

\subsubsection{Distance Vector}

Wireless Routing Protocol [3]. It is a table-based protocol aiming to maintain routing information among all nodes in the network. Update messages are periodically exchanged only between neighboring nodes and contain a list of update information such as the destination, the distance to the destination, and the second-to-last hop to the destination. Nodes do not exchange the whole distance vector table information, rather they exchange tuples that reflect link changes. If no changes occur, they only transmit Hello messages to maintain neighbor information. By maintaining predecessors of destinations it is able to recursively detect loops.

Destination Sequence Distance Vector [4]. This protocol augments the classical, distributed Bellman-Ford by tagging each distance entry dik $(\mathrm{j})$ by a sequence number that originated in the destination node i. Each node maintains this sequence number, incrementing it each time the node sends 
an update to the neighbors. The sequence number is disseminated in the network via update messages. The destination sequence number is used to determine the "freshness" of a route. Always the latest sequence number is used for updating routes. For equal sequence numbers, the one with the smallest distance metric is used. It has been shown that DSDV avoids long-lived loops and counting to infinity problems.

\subsubsection{Reactive Protocols}

These protocols are also referred to as on-demand routing protocols, because nodes initiate route discovery via a request/ reply mechanism, only if the need to route a packet to a specific destination is present. As an optimization they, maintain a cache of soft-state route entries for future use.

Temporally Ordered Routing Algorithm, TORA [5]. was designed to discover routes on demand, provide multiple routes to a destination, establish routes quickly, and minimize communication overhead.

Dynamic Source Routing, DSR [5]. It uses source routing, with each packet carrying in its network layer header the complete ordered list of the nodes it will pass. Routes are resolved through a flood based route discovery process during which the path is recorded in the control packets. The on-demand nature of the protocol eliminates the need for periodic updates and neighbor discovery beacons.

Ad Hoc On Demand Distance Vector, AODV [6]. It builds on DSDV's sequence number mechanism. Sequence numbers of control packets are used to ensure that paths are loop free and recent. Intermediate nodes update their forwarding tables during the reply phase of the route discovery. The back up routing mechanism of AODV-BR [7] provides resilience to frequent topology changes.

\subsubsection{Zone-based Clustered Protocols}

Zone Routing Protocol [8]. It is a zone or cluster-based routing protocol that combines the best of proactive and reactive routing protocols. Zone is an area within a specified range. Its operation is bimodal, utilizing proactive routing for intra-zone communications and reactive routing across zones. If the route to a node is not known, the request is broadcast to the zone perimeter and from that point further an on-demand protocol is used to establish the route.

\subsubsection{Location Aware Protocols}


Location Aided Routing [9]. This complementary protocol employs explicit location information to improve routing performance of on demand routing protocols. It enhances the flooding phase of the route discovery using location information.

\subsection{Table Driven and On-Demand Ad Hoc Protocols}

Two different types of routing protocols: table driven link state protocol and source initiated on-demand routing protocol [10]. Table driven link state protocols where each node gathers information about the state of the links that are available and keep them in tables. The costs of the outgoing links are updated in these tables. Some of the transferred information could be outdated due to the propagation delays. These protocols require high bandwidth to keep links status information current. Some linkstate protocols reduce the bandwidth by minimizing the transfer of state link information. They distribute the information only to the affected nodes.

On-demand protocols do not gather or distribute information unless there is a need to establish communication. There are no tables to maintain, and no data update is required. These protocols are efficient for Ad hoc networks since they minimize the overhead of routing.

This paper presents in section.2 an overview of routing protocols over mobile ad hoc networks like DSR, AODV and HDSR, section.3 presents Optimization of HDSR, section. 4 presents the simulation model and results and finally section. 5 presents the conclusion and the future work.

\section{2-Previous and related Work}

Due to nodes mobility, the topology of an ad hoc network may change rapidly and unpredictably over time. The design of network protocols for MANET is a complex issue, these networks need efficient distributed algorithms to determine network organization (connectivity), link scheduling and routing.

Most routing protocols for mobile ad hoc networks, such as: Ad Hoc on Demand Distance Vector Protocol (AODV), Dynamic Source Routing (DSR) are designed without explicity considering quality of service of the generated route [11]. These routing protocols provide the capability for establishing minimum hop paths between nodes on a best effort basis regardless of QoS [12]. In our work, we analyze the performance of these protocols and we present an efficient load-balancing scheme for supporting QoS over MANET that allows nodes to:

-Distribute and efficiently use network resources (buffer space), 
-Reduce network congestion by change route, -Increase overall performance (throughput).

\subsection{Hierarchical Dynamic Source Routing( HDSR)}

In Hierarchical Dynamic Source Routing (HDSR) [13] it classifies the participating nodes of the network as Mobile Nodes (MN) and Forwarding Nodes (FN). It assigns different functionalities to those nodes depending on what type of node they are. MNs initiate route discovery. FNs help them to find source route to the destination MN. The destination $\mathrm{MN}$ replies back through the FNs to source MN. Once source MN discovers the routes, it starts sending packets to the destination. FNs assist the MN to forward packets to destination MN. Route discovery and route maintenance in this technique are different from those in DSR. When a source MN originates packet to a destination MN. If the source cannot find a source route in its route cache, it initiates a route discovery by transmitting a "route request packet" as a local broadcast packet. Only FNs, which are within the range of the source MN receive the broadcast packet. Other MNs, which are also within the range of source $\mathrm{MN}$ and which are not the destination of this packet, discard the broadcast message and do not broadcast further. Only the FNs re-broadcast the request to other FNs unless the destination MN receives this route request packet. The destination MN then replies back to the source MN through the FNs. After receiving the route reply, the source MN record the source route in its cache and starts sending packets to the destination $\mathrm{MN}$ using the source route it has just discovered.

Route maintenance is performed by FNs only. When a FN detects that the next link from itself to the next MN or FN is broken, it updates its own route caches by marking all the paths which use the broken link as invalid and sends route error message to the source $\mathrm{MN}$ and all other FN which use the broken link for packet transmission. We will explain now how it reduces overhead packet during the route discovery processes and prevent route request and route reply flooding.

\section{FORWARD NODE (FN) SELECTION MECHANISM}

We observed that increasing the number of FNs in the network improves the throughput up to a certain point [13]. After that point (9-11 FNs), increasing the number of FNs will increase the routing overhead and degrade the performance. So, 
efficient and adaptive FNs selection mechanisms is important for optimization of network performance. In this section, route discovery strategies is proposed by incorporating self-selection into the rebroadcast process. The use of self-selection enables intermediate nodes to make effective localized rebroadcast decisions about whether or not the FN participate in a route discovery. So, controlling the number of the FNs. In this process, the source node $\mathrm{MN}$ is responsible for specifying a required metric in each RREQ packet. All nodes that do not meet this metric may then elect not to participate in route discovery. To illustrate simply the benefits of self-selecting strategies, assume a node $\mathrm{S}(\mathrm{MN})$ (Figure 1) is required to discover a route to node $\mathrm{D}$ $(\mathrm{MN})$ without any prior knowledge (e.g. hop count, location information). Now, assume each node maintains a utility function, U, (e.g. based on mobility, topology and power). To minimize the number of route request retransmissions, we can modify the route discovery procedure to allow the nodes (FNs) with the highest levels of utility to rebroadcast in the first

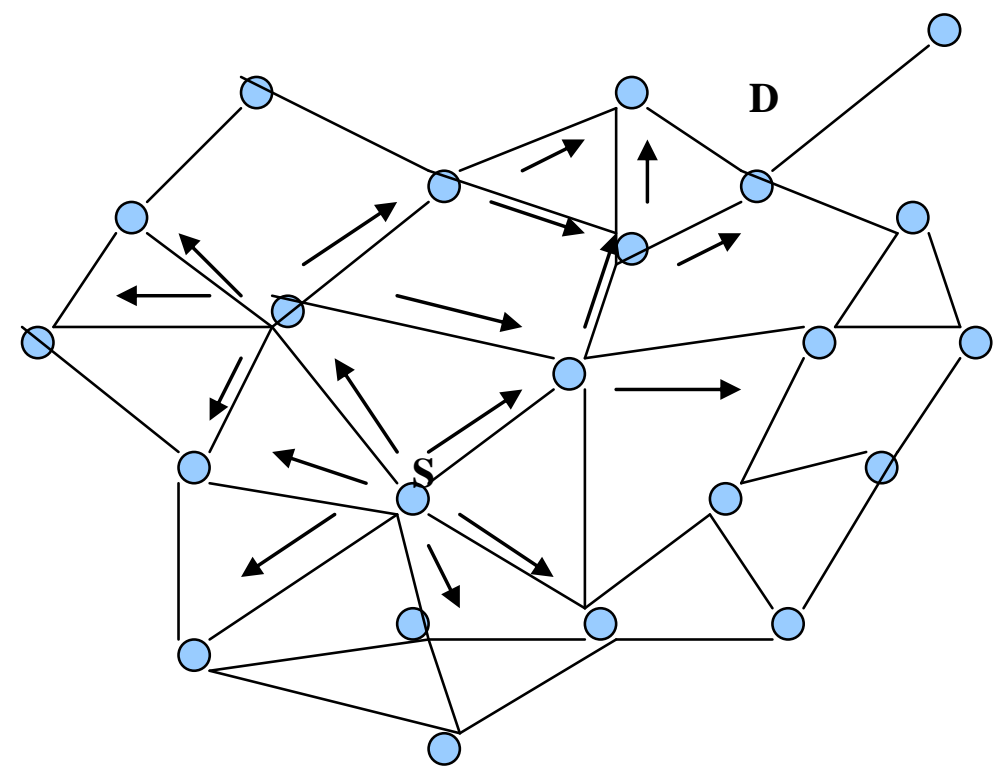

Fig. 1. Utility-based Self-Selection

route discovery attempt.Lets assume in this case, only nodes (FNs) with a utility level greater than 4 may rebroadcast. In this scenario, only five nodes (FNs) rebroadcast whereas using a pure flooding approach 21 nodes may rebroadcast. Hence, a reduction of 17 nodes is achieved. In networks with high node density and traffic, such strategies may significantly improve data throughput and allow each node to conserve resources 
if required. The idea of using mobility to minimize the number of control packets was introduced in DREAM [14]. DREAM is a proactive routing strategy, which optimizes the frequency at which route updates are sent by nodes to the speed at which they travel. Therefore, the nodes, which travel at high speeds send update packets more frequently. In this strategy, we use mobility to reduce route discovery redundancy in on-demand routing. To do this, we modify the route discovery strategy to restrict the RREQ rebroadcasts packets to occur over more stationary nodes first and then reducing the number of FNs. A utility function is introduced, which determines a maximum allowable node speed during each route discovery phase. Therefore, only nodes which are traveling at a lower speed than the one specified in the utility function will rebroadcast. The benefits of this strategy include:

- Increased route stability over blind flooding as selecting least mobile nodes (FNs) results in fewer route failures.

- Reduction in Broadcast Storm Problem due to fewer rebroadcast nodes (FNs) during route discovery.

- Total number of control packets may be reduced significantly, especially in dense networks.

In the following proposed algorithms, $\mathrm{P}$ is used to vary the utility functions, which is used to limit the number of re-broadcasting nodes (FNs). In our study we used five different values for $\mathrm{P}$ to investigate the effectiveness of the self-selecting strategies. In future studies, we plan to study how adjusting $\mathrm{P}$ according to known levels of mobility and reachability at each node influences performance. The algorithm is outlined below:

1. RREQmax $\leftarrow$ Maximum number of route request retries

2. FNmax $\leftarrow$ Maximum number of forwarding nodes

3. Vmax $\leftarrow \tau$ Maximum speed at which a node can travel

4. $\mathrm{Vu} \leftarrow$ Maximum allowable node speed

5 . VNoMax $\leftarrow$ Flag used for pure flooding

6 . $\mathrm{P} \leftarrow\{0.125,0.25,0.5,0.75,1.0\}$ (* Used to select different

speed levels $*$ )

7. FNmax $\leftarrow 11$

8. RREQmax $\leftarrow 6$

9. for $\mathrm{j} \leftarrow 0, \mathrm{j} \neq$ FNmax, $\mathrm{j}++$ 
8. for $\mathrm{i} \leftarrow 0, \mathrm{i} \neq \mathrm{RREQmax}, \mathrm{i}++$

9. $\mathrm{Vu} \leftarrow \mathrm{Vmax}$.Pi

10. Forward RREQ(Pi, Vu)

11. wait for reply

12. if Route $=$ found

13. initiate data transmission

14. break loop

15. endif

16. endfor

17. endfor

18. if Route $=$ notfound

19. Forward RREQ(0,VNoMax)

20. wait for reply

21. if Route $=$ found

22. initiate data transmission

23. else

24. return route not found

25. endif

26. endif

In this algorithm, the source node $(\mathrm{MN})$ begins by calculating the mobility utility function $(\mathrm{Vu})$, which selects a value for maximum allowable velocity at each intermediate node during a route discovery phase. This value is then passed to the Forward Node and the Forward RREQ functions where it is attached to the RREQ packet and disseminated to the network. When an intermediate node receives a RREQ packet and it does not have a route to the required destination, it checks to see if its current speed (obtained via GPS) is less than $\mathrm{Vu}$. If yes, then it will rebroadcast the RREQ packet. Note that, we have selected 5 different mobility levels (defined in P), which are used to increase $\mathrm{Vu}$ when a route discovery fails to determine a route. If a route is still not found, then a final route discovery is initiated, which allows all nodes to rebroadcast resulting in a Blind flood. 


\section{SIMULATION MODEL AND RESULTS}

Simulation is used to implement and test the performance of these protocols. The key parameters and results are summarized in tables $1 \& 2$ below.

Table 1

Simulation Parameters

\begin{tabular}{|l|l|}
\hline Parameter & Value \\
\hline Transmission Range & 250 Meters \\
\hline Medium Access Control (MAC) & IEEE802.11 \\
\hline Raw Capacity & $\mathrm{kb} / \mathrm{s} 2$ \\
\hline Traffic Sources(CBR) & $512 \mathrm{~b} / \mathrm{s}$ \\
\hline Mobility Model & Waypoint model \\
\hline Speed & $0-20 \mathrm{~m} / \mathrm{s}$ \\
\hline
\end{tabular}

Table 2

Simulation Results

\begin{tabular}{|l|l|l|l|}
\hline Performance metrics & \multicolumn{1}{|c|}{ DSR } & \multicolumn{1}{|c|}{ AODV } & \multicolumn{1}{|c|}{ HDSR } \\
\hline Packet delivery fraction (\%) & 56.88 & 83.66 & 90.48 \\
\hline Average delay (s) & 1.36 & 0.26 & 0.14 \\
\hline Throughput (kbps) & 100 & 170 & 200 \\
\hline Total Capacity (kbps) & 500 & 600 & 800 \\
\hline $\begin{array}{l}\text { MAC Control Capacity } \\
\text { (kbps) }\end{array}$ & 600 & 470 & 520 \\
\hline
\end{tabular}

\section{A. Effect of mobility}

Figures $(2,3,4)$ show performances of simulations of $80 \mathrm{MNs}$ scenario versus the pause time of mobile nodes. The size of the rectangular area that mobile nodes are located in 1000x1000 meters. There are 20 CBR sources with data packet rate of 2 packets per seconds, 12 FNs in additions to MNs and locations of the FNs are chosen randomly as well. (Figure 2) shows the routing overhead of the protocols. The routing overhead in the (HDSR) technique is consistently lower than DSR in all scenarios, and for this scenario it is approximately 50 percent lower. We observed that overhead improvement in this technique is higher when the number of nodes in the networks grows. The 
difference between this technique and DSR overhead increases when the mobility is higher (i.e., shorter pause times). Due to the higher number of routing overhead packets, the network with DSR routing protocol has lower bandwidth for data packets, which we think adversely affects performance metrics in DSR compared with this technique. For example, throughput of the network is improved 3 times in high mobility and 20-30 percent in low mobility cases compared with that of DSR (Figure 3). In different scenarios, the throughput is always better with HDSR. The average endto-end delay is also improved. (Figure 4) shows average end-to-end delay of scenario with 80 mobile nodes. In that case, the delay is 3 times higher than that for very high mobility (i.e. pause time less than 50 seconds) and few tens of times in low mobility cases. Delivery ratio was better than DSR too. (Figure 2) shows how this technique saves overhead which results better throughput (Figure 3). Number of FNs in the network naturally affects the performance of this technique. We observed that increasing the number of FNs in the network improves the throughput up to a certain point. That is why we think that distribution of FNs in the network is important for optimization of the performance figures, and we will consider this point in the future work. We consider an example scenario corresponding to a network of 100 nodes with zero pause time (constant mobility).

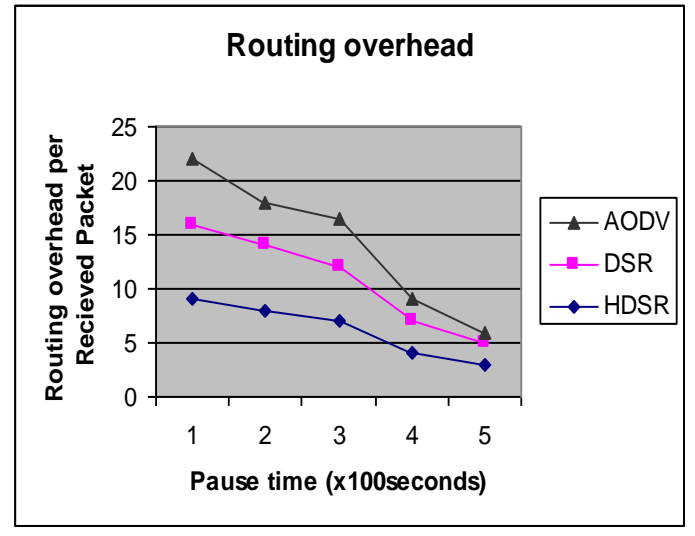

Fig. 280 MN Scenario

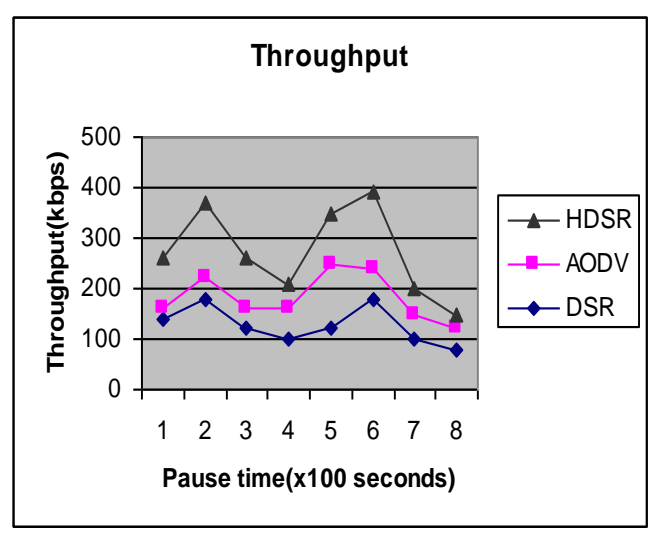

Fig.3 80 MN Scenario 


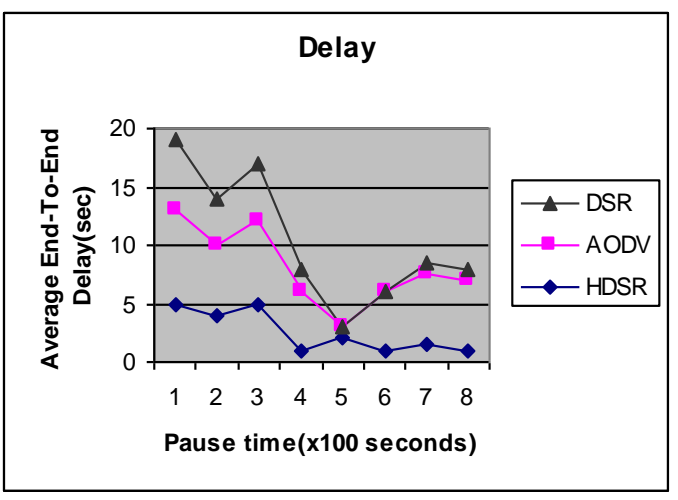

Fig.4 80 MN Scenario

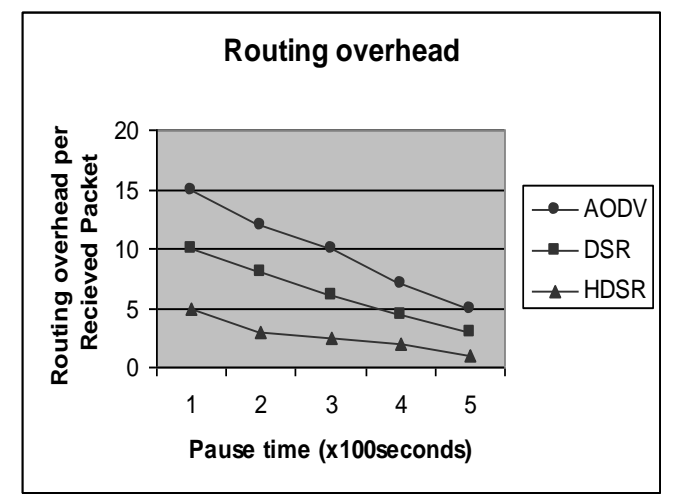

Fig.5 50 MN Scenario

Traffic in this example involves $40 \mathrm{CBR}$ sources each generating packets at the rate of $2 / s$, each of size 512 bytes. For this example, the application-oriented metrics point out that DSR has a nearly 32 percent lower delivery fraction than AODV and 5 percent higher delay. But for HDSR, DSR has a nearly 45 percent lower delivery fraction than HDSR and few 10 percent higher delay.

\section{B. Effect of number of nodes}

We calculated the routing overhead versus the pause time of mobile nodes for each protocol at 50 MNs scenario. From (Figure $2 \& 5$ ) we can observe that as the number of mobile nodes decreases the routing overhead per received packet decreases. In addition, We calculated the throughput versus the pause time of mobile nodes for each protocol at 50 MNs scenario. From (Figure $3 \& 6$ ) we can observe that as the number of mobile nodes decreases the throughput increases. Moreover, we can find that how HDSR saves overhead in a 50 mobile node scenario which results in better throughput. We can conclude from the above observations that HDSR limits the number of nodes that participate in the route discovery of the protocol, which in turn reduces overhead and delay compare to DSR and AODV. That architectural change provides HDSR to reduce the routing overhead significantly because number of nodes that involve in the route discovery is smaller and they can find and return routes faster to the source. That can reduce the end to end delay too. In addition to those, MNs do not need to acquire and maintain any statistical information about the neighbors or do not need to send maintenance messages or location information about the neighbors. 
Such reductions could significantly save bandwidth of the network, hence improve the throughput of the network.

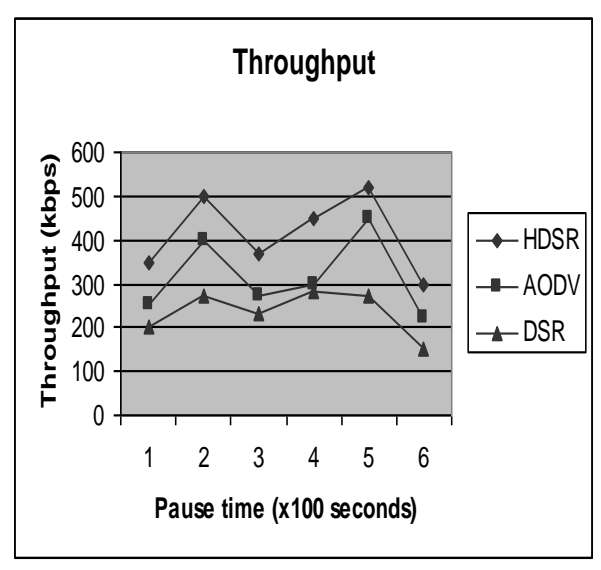

Fig. $650 \mathrm{MN}$ Scenario

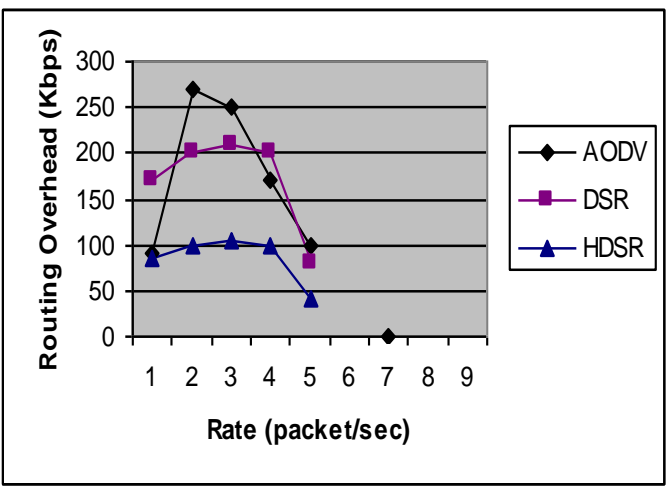

Fig. 8 Routing overhead capacity versus versus rate

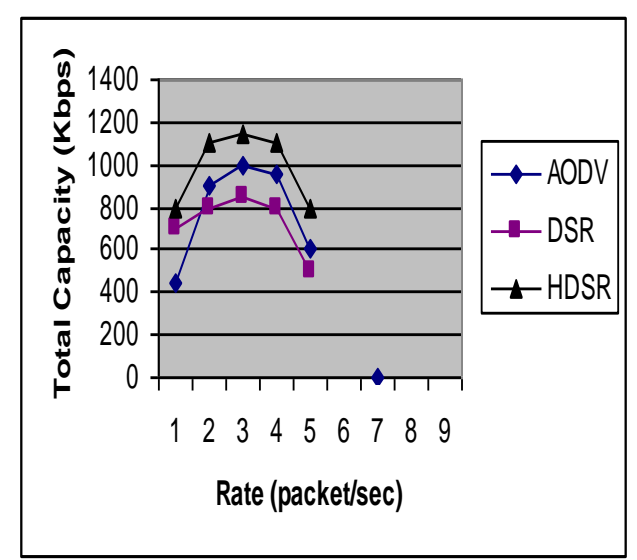

Fig. 7 Channel capacities versus rate

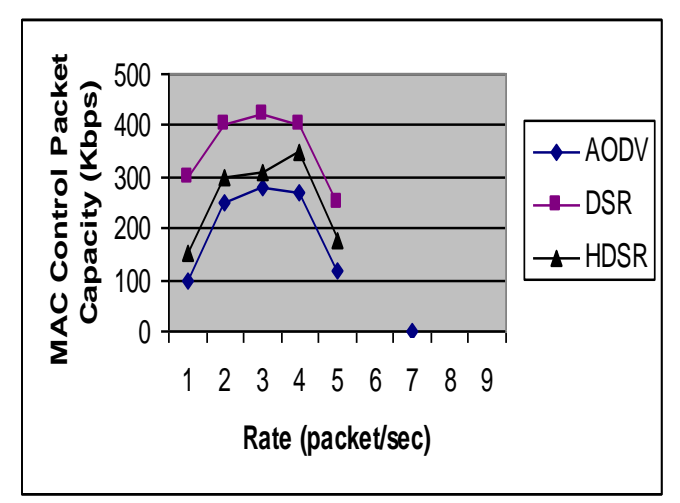

Fig. 9 MAC control packets capacity versus rate

\section{Effect of loading}

We calculate the channel capacity of each protocol versus packet rate. (Figure 7) Moreover, we can observe that the capacity decreases at very high rates. We can 
observe that at low rate the routing overhead of AODV is lower than the routing overhead of DSR, the contrary is true as the rate increases until eight packets/sec. We can explain this as follows: at low rates, DSR generates more replies and errors (gratuitous or other wise). whereas at high rates, AODV generates more route request packet, which increases the routing overhead. Moreover, (Figure 9) shows MAC control packet capacity with respect to packet rate. We can observe that DSR has a larger number of MAC packets than AODV. This is a result of the large number of RTS retransmissions due to collisions or link failures in DSR. In addition, we can observe from (Figure $8 \& 9$ ) that routing overhead and MAC control packet represent $40 \%$ from total capacity in AODV while they represent $70 \%$ from total capacity in DSR. Therefore, data packets using AODV routing protocol utilize channel capacity with a percentage higher than the DSR. This gives the superiority to AODV as a routing protocol with efficient utilization of the channel capacity with respect to the offered load. Finally, (Figure 10) shows MAC control packets capacity into the network capacity with variable pause time. We can observe that DSR has a larger number of MAC control packets than AODV. This is a result of the large number of RTS retransmissions due to collisions or link failures.

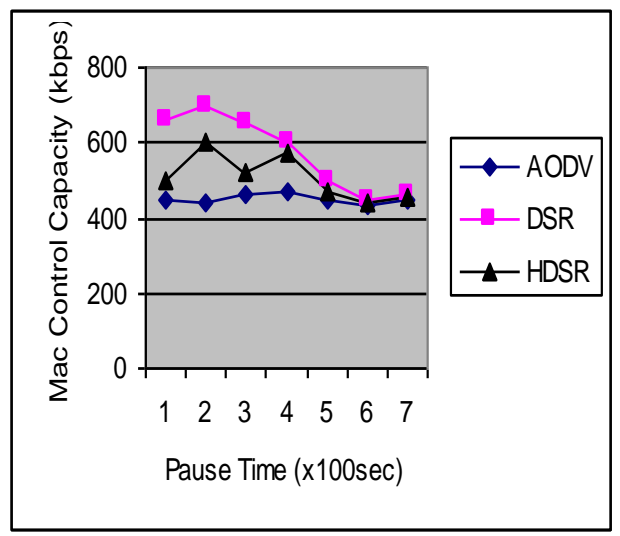

Fig. 10 MAC control packets

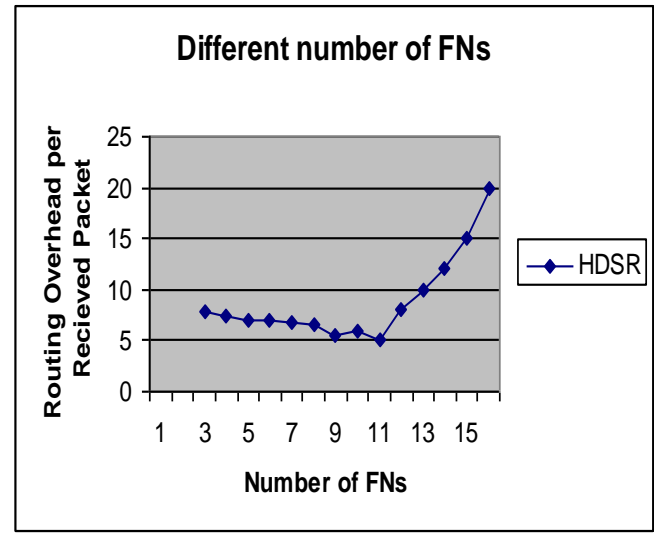

Fig. $1150 \mathrm{MN}$ Scenario

utilization versus mobility 


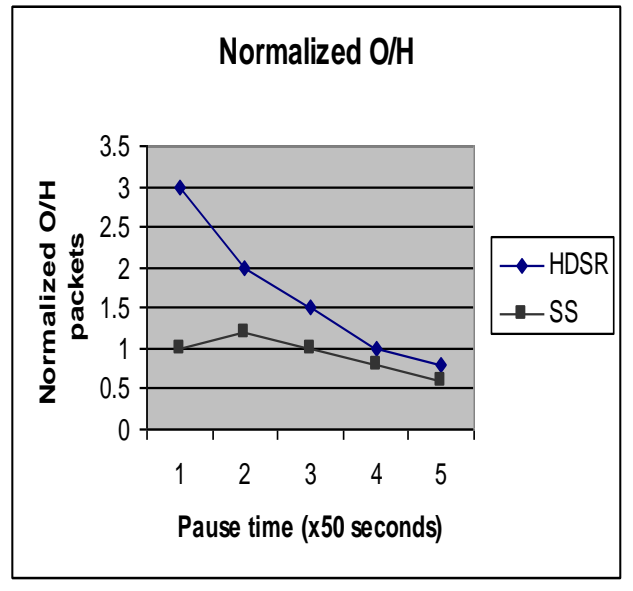

Fig. 12100 Node Scenario

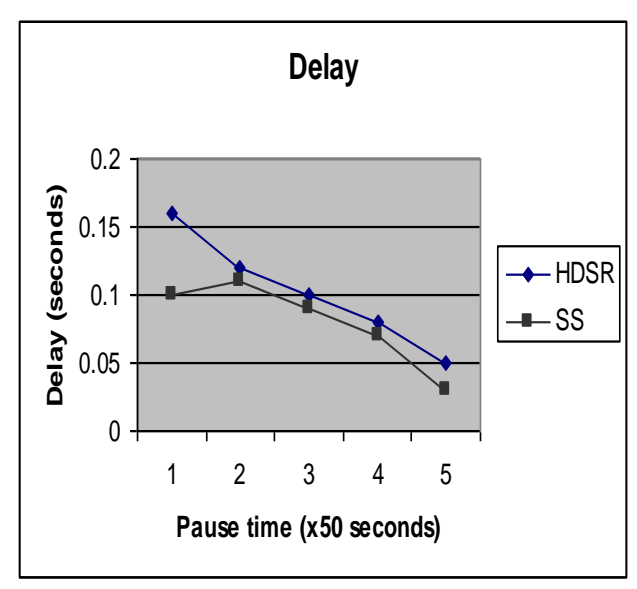

Fig. 13100 Node Scenario

\section{Effect of number of FNs}

Number of FNs in the network naturally affects the performance of HDSR. We can observe that increasing the number of FNs in the network improves the throughput up to a certain point. After that point (9-11 FNs), increasing the number of FNs will increase the routing overhead and degrade the performance (Figure 11) [13]. (Figure 12) illustrate the normalized control overhead versus pause time introduced into the network for 100 node simulation. We have shown that Self-selecting strategies (SS) produce significantly lower normalized control overheads than HDSR. (Figure 13) illustrate the Delay versus pause time introduced into the network for 100 node simulation. We have shown that Self-selecting strategies (SS) produce significantly lower delay than HDSR. We conclude that distribution of FNs in the network is important for optimization of the performance figures.

\section{CONCLUSIONS}

We have compared the performance of DSR and AODV, two prominent ondemand routing protocols for ad hoc networks. DSR and AODV both use on-demand route discovery, but with different routing mechanics. In particular, DSR uses source routing and route caches, and does not depend on any periodic or timer-based activities. DSR exploits caching aggressively and maintains multiple routes per destination. AODV, on the other hand, uses routing tables, one route per destination, and destination sequence numbers, a mechanism to prevent loops and to determine 
freshness of routes. We observed that these routing protocols provide the capability for establishing minimum hop paths between nodes on a best effort basis regardless of QoS.

In Hierarchical Dynamic Source Routing (HDSR) which is an efficient technique for supporting QoS in Mobile Ad Hoc Networks. This technique is able to improve network performance figures, namely routing overhead, end-to-end delay, total capacity, MAC control packet capacity and throughput significantly. We have shown also that distribution of Forward Nodes (FN) in the network is important for optimization of the performance figures. We present an efficient and adaptive FN selection mechanism for HDSR as well as optimization of number of FNs in a given scenario. Our future work is to define a new parameters to provide load balancing, support fault tolerance, and select optimal routes.

\section{REFERENCES}

[1]G.Pei, M.Gerla, and T. W.Chen, Fisheyestate routing in mobile ad hoc networks, Proceedings of the 2000ICDCSWorkshops, Taipei, Taiwan, Apr. 2000, pp D71D78.

[2]P.Jacquet, P.Muhlethaler, and A.Qayyum, Optimized link state routing protocol, IETF MANET, Internet Draft, Nov.1998.

[3]S.Murthy and J.J.Garcia-Luna-Aceves, An efficient routing protocol for wireless networks, ACM Mobile Networks and Applications Journal, Special issue on Routing immobile Communication Networks, 1996.

[4]C.E.Perkin and P.Bhagwat, Highly dynamic destination sequenced Distance vector routing (dsdv) for mobile computers, ACMSIGCOMM: Computer Communications Review, vol.24, no.4, pp.234-244, October 1994.

[5]D.B. Johnson and D.A. Maltz, the dynamic source routing protocol for mobile ad hoc networks, Mobile Computing, edited by Tomas Imielinskiand HankKorth, Kluwer AcademicPublishers,ISBN:0792396979,1996, Chapter pages 153-181.

[6]C.E.Perkins and Royer, Ad-hoc on-demand distance vector outing, second IEEE Workshop on Mobile Computing Systems and Applications, pp.90-100, February1999.

[7]S.J.Lee and M.Gerla, Backup routing in aodv,in Infocom 2003. 
[8]Z.Haas and M.zone routing protocol (zrp) for ad hoc networks, IETF Internet Draft,Version4,July,2002.

[9]Y.Ko and N.H.Vaidya,.Location aided routing (lar) mobile ad hoc networks,.MOBICOM1998.

[10] Chrles E.Perkins,The Ad Hoc Network,Boston,2001.

[11] M. K. Ahmed, O. M. El-Ghandour, H. R. Soliman. Comparative Evaluation of routing Protocols over Mobile Ad Hoc Network (MANET). Journal of Engineering Science (JES), Faculty of Engineering, Assiut University, pp. 843856, vol.34-no.3, May2006.

[12] M. K. Ahmed, O. M. El-Ghandour, H. R. Soliman. A New Efficient Scheme for supporting Qos over Mobile Ad Hoc Network (MANET). The2006 International Workshop on System-On- Chip(IWSOC), Dec27-29, 2006, Cairo , Egypt.

[13] Kemal E. Tepe, Mohamed Naserian, and Tarique Mohamed, " A New Hierarchical Routing Protocol, Hierarchical Dynamic Source Routing, for Ad Hoc Wireless Networks" Proc. 14th Wireless Conference, Calgary, 2004.

[14] S. Basagani, I. Chlamtac, V.R. Syrotivk, and B.A. Woodward. A Distance Effect Algorithm for Mobility (DREAM). Proc. 4th Annual ACM/IEEE International Conference on Mobile computing and Networking (Mobicom 98), Dallas, TX, 1998.

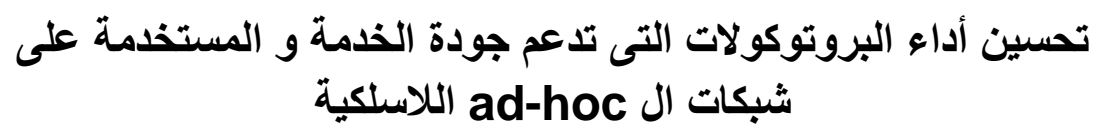

تعتبر شبكات ال ad-hoc اللسلكية شبكات حديثة نوعا ما و أصبح لها وجود فى مجال البحث العلمى. تتميز هذه الثبكات بعدم وجود شكل أو تتظبم ثابت لها، حيث أنها شبكات ذاتيـة التتظيم. وقد ظهر العديد من البروتوكولات المستخدمة فى هذه الثـبكات والتى تخنلـف فـى إنجازاتهـا ومـدى تعقدها. أغلب هذه البروتوكولات تـم تصميمها لإيجاد أقصر مسـار لنقل البيانات دون مراعاة مستوى جودة الخدمة. يقوم هذا البحث بتحليل أداء هذه البروتوكولات عن طريق دراسة مجموعة من المعابير كما يقوم بعرض تكنيلك فعال بدعم جودة الخدمة على هذه الثبكات. بالإضافة إلى تحسين أداء هذا التكنيك. 